LAWRENCE LIVERMORE N A T IO N A L LABORATORY

\section{The Barenblatt Turbulent Burst Problem in Kull}

M. Ulitsky

June 1, 2005 
This document was prepared as an account of work sponsored by an agency of the United States Government. Neither the United States Government nor the University of California nor any of their employees, makes any warranty, express or implied, or assumes any legal liability or responsibility for the accuracy, completeness, or usefulness of any information, apparatus, product, or process disclosed, or represents that its use would not infringe privately owned rights. Reference herein to any specific commercial product, process, or service by trade name, trademark, manufacturer, or otherwise, does not necessarily constitute or imply its endorsement, recommendation, or favoring by the United States Government or the University of California. The views and opinions of authors expressed herein do not necessarily state or reflect those of the United States Government or the University of California, and shall not be used for advertising or product endorsement purposes.

This work was performed under the auspices of the U.S. Department of Energy by University of California, Lawrence Livermore National Laboratory under Contract W-7405-Eng-48. 


\section{The Barenblatt Turbulent Burst Problem in Kull Mark Ulitsky AX-Division L-023}

Significant changes have recently been made to the way in which explicit diffusion is carried out for the turbulent mix model in Kull. That is, the upwind method of calculating divergences of drift/diffusion velocities has been replaced by a more standard treatment, where diffusion coefficients are averaged to the nodes, multiplied by the gradient of some field, and then the usual divergence of this product is taken. Boundary conditions are handled very differently in these two approaches, and for Rayleigh-Taylor problems, the upwind method leads to non-monotonic density profiles and an incorrect treatment of fluxes at mesh boundaries. The more standard approach to diffusion is also taking advantage of the new, more powerful region definition in Kull which puts buffer zones around the region at material boundaries (external zones) and also at mesh boundaries (external surface zones). This allows for much more control of the fluxes at these boundaries, and also saves considerable computer time and memory, since we can now take gradients and divergences of region based fields (rather than the old approach of creating a mesh based field to store the region quantity, performing the differential operators on the mesh based field, and finally storing the mesh based field back in the region variable).

To verify that the new diffusion algorithm is working as anticipated, we have selected a nonlinear diffusion-dissipation problem known as the Barenblatt turbulent burst problem. This problem involves solving two coupled nonlinear partial differential equations (a very simplified k- $\varepsilon$ model) for the temporal and spatial evolution of the turbulent kinetic energy $(\mathrm{k})$ and the turbulent dissipation rate $(\varepsilon)$. What makes this nontrivial problem useful from a verification standpoint is that it has an analytic selfsimilar solution.

The coupled equations for the Barenblatt problem are:

$$
\begin{aligned}
& \frac{\partial k}{\partial t}=\frac{\partial}{\partial x}\left(D \frac{\partial k}{\partial x}\right)-\varepsilon \\
& \frac{\partial \varepsilon}{\partial t}=\frac{\partial}{\partial x}\left(D \frac{\partial \varepsilon}{\partial x}\right)-C_{\varepsilon 2} \frac{\varepsilon^{2}}{k} \\
& D=C_{\mu} \frac{k^{2}}{\varepsilon}
\end{aligned}
$$

In Kull, the EZturb k- $\varepsilon$ model is tightly coupled to the Lagrange hydro, and so the actual mix model equations we solve when the model is active are:

$$
\frac{d \alpha_{r}}{d t}=\frac{\partial}{\partial x_{j}}\left(\frac{\mu_{t}}{\sigma_{M}} \frac{\partial}{\partial x_{j}}\left(\frac{\alpha_{r}}{\rho}\right)\right)
$$




$$
\begin{aligned}
& \frac{d \alpha_{r} \rho_{r}}{d t}=\frac{\partial}{\partial x_{j}}\left(\frac{\mu_{t}}{\sigma_{M}} \frac{\partial}{\partial x_{j}}\left(\frac{\alpha_{r} \rho_{r}}{\rho}\right)\right) \\
& \frac{d \rho u_{i}}{d t}=-\frac{\partial \tau_{i j}}{\partial x_{j}} \\
& \frac{d \alpha_{r} \rho_{r} I_{r}}{d t}=\frac{\partial}{\partial x_{j}}\left(\frac{\mu_{t}}{\sigma_{U}} \frac{\partial}{\partial x_{j}}\left(\frac{\alpha_{r} \rho_{r} I_{r}}{\rho}\right)\right)+P_{r}^{I}+\delta_{I, D i s s} \alpha_{r} \rho_{r} \varepsilon \\
& \frac{d \rho k}{d t}=\frac{\partial}{\partial x_{j}}\left(\frac{\mu_{t}}{\sigma_{K}} \frac{\partial k}{\partial x_{j}}\right)-\tau_{i j} S_{i j}-\rho \varepsilon+P \\
& \frac{d \rho \varepsilon}{d t}=\frac{\partial}{\partial x_{j}}\left(\frac{\mu_{t}}{\sigma_{z}} \frac{\partial \varepsilon}{\partial x_{j}}\right)-C_{1 \varepsilon} \tau_{i j} S_{i j} \frac{\varepsilon}{k}-C_{2 \varepsilon} \rho \frac{\varepsilon^{2}}{k}+C_{3 \varepsilon} P \frac{\varepsilon}{k}
\end{aligned} .
$$

Here, $\alpha_{r}, \rho_{r}$, and $\mathrm{I}_{\mathrm{r}}$ are the volume fraction, thermodynamic density, and specific internal energy (by mass) for material r. $S_{\mathrm{ij}}$ is the strain rate tensor, and $\tau_{\mathrm{ij}}$ is the turbulent shear stress tensor, for which we use the following Boussinesq approximation:

$$
\tau_{i j}=\delta_{\text {Iso }} \frac{2}{3} \rho k \delta_{i j}-\delta_{\text {Anso }} 2 \mu_{t}\left(S_{i j}-\frac{\delta_{i j}}{3} \frac{\partial u_{k}}{\partial x_{k}}\right) .
$$

The turbulent viscosity includes the effects of both shear and buoyancy and takes the form:

$$
\mu_{t}=C_{\mu} \rho \frac{k^{2}}{\varepsilon}+C_{\omega} \rho \frac{k^{3}}{\varepsilon^{2}} \sqrt{\omega^{2}+\frac{\nabla p \cdot \nabla p}{p \rho}-\frac{\nabla p \cdot \nabla \rho}{\rho^{2}}[1-\Theta(\nabla p \cdot \nabla \rho)]} .
$$

The unlimited form of the buoyant production term is given by:

$$
P=-\frac{\mu_{t}}{\sigma_{\rho} \rho^{2}} \nabla p \cdot \nabla \rho
$$

and the way this term manifests itself in the internal energy equation is: 
$P_{r}^{I}=-h_{r} p_{r} \nabla \cdot\left(\frac{\mu_{t}}{\sigma_{\rho} \rho^{2}} \nabla \rho\right)$

The model constants are $\mathrm{C}_{1 \varepsilon}, \mathrm{C}_{2 \varepsilon}, \mathrm{C}_{3 \varepsilon}, \sigma_{\mathrm{M}}, \sigma_{\mathrm{U}}, \sigma_{\mathrm{K}}, \sigma_{\mathrm{Z}}, \sigma_{\rho}, \mathrm{C}_{\mu}$, and $\mathrm{C}_{\omega}$. Also, $\delta_{\text {Iso }}, \delta_{\text {Aniso, }}$, and $\delta_{\mathrm{I} \text {,diss }}$ are on/off switches that can be set to 1 or 0 .

At this point, it may not be obvious how this form of the EZturb model reduces to what must now appear to be an extremely simplified turbulent model, namely, the Barenblatt burst model. However, if we set the three $\delta$ switches to 0 , then $\tau_{\mathrm{ij}}$ will be identically zero (we therefore eliminate any coupling of the mix model to the mean flow) and we will also eliminate dissipation in the internal energy equations. If we then set $\sigma_{\mathrm{M}}$, $\sigma_{U}$, and $\sigma_{\rho}$ to very large values, we will prevent any diffusion of volume fraction, mass, and internal energy for all materials, as well as kill all the buoyant production terms. Finally, if we initialize the mean velocity to zero, the mean density to unity, and set $\mathrm{C}_{\omega}=$ 0 , then the EZturb model will reduce exactly to the Barenblatt model, and we can run Kull in the usual fashion (take a Lagrange hydro step followed by a mix step using operator splitting).

If $\mathrm{C}_{\varepsilon 2}=2$, the solution to equations 1,2 takes the simple form of:

$$
\begin{aligned}
& k(x, t)=\xi_{0}^{2} t^{-4 / 3}-\frac{x^{2} t^{-2}}{6 C_{\mu}} \\
& \varepsilon(x, t)=\frac{k(x, t)}{t} \\
& \xi_{0}^{2}=\frac{D_{0} t_{0}^{1 / 3}}{C_{\mu}} .
\end{aligned}
$$

Clearly, the analytic solution "blows up" at $t=0$, and so we will use $t_{0}=.1 \mathrm{sec}$. The diffusion constant $\mathrm{D}_{0}$ will be set to $.01 \mathrm{~cm}^{2} / \mathrm{sec}$, and $\mathrm{C}_{\mu}$, the von Karaman constant, will take the standard value of .09. For the spatial domain, we will consider four meshes (25 zones, 50 zones, 100 zones, and 200 zones) in which $-.15 \leq x \leq .15$.

Figure 1 shows comparisons of the numerical and analytic solutions for $\varepsilon(\mathrm{x}, \mathrm{t})$ for $\mathrm{t}$ $=\mathrm{t} 0$ (.10 sec), $.15 \mathrm{sec}, .20 \mathrm{sec}$, and $.4 \mathrm{sec}$ for the mesh with the coarsest zoning. From the figure, it is evident that even with only 25 zones, the agreement between the solutions is quite good. The initial profile for e is parabolic and the coarseness of the grid manifests itself at the intercepts of the parabola with the $\mathrm{x}$-axis. This mismatch gets better as the zoning is refined. Also, the analytic solution needs to be cut off at the diffusion front. That is, there is nothing mathematically unreasonable about having $\mathrm{k}$ and $\varepsilon$ take on 
negative values; however, it is unphysical for kinetic energy or dissipation to be negative. Therefore, a simple " $\varepsilon=\max (0, \varepsilon)$ " is used when presenting the analytic solutions. Having a hard zero in the code can be problematic, and so floor values of $1.0 \mathrm{e}-14$ and $1.0 \mathrm{e}-16$ are used for $\mathrm{k}$ and $\varepsilon$ respectively. Figures 2, 3, and 4 are the same as Figure 1, except that we are looking at the effect of increasing the mesh resolution.

From the previous figures, it is difficult to get a feel for the error committed by the numerical solution of the PDEs. To remedy this fact, we can compute a relative error defined as $\left|\varepsilon_{\text {analytic }}-\varepsilon_{\text {numerica }} \mathrm{l}\right| / \varepsilon_{\text {numerical }}$ and plot this error as a function of position at multiple times for the different mesh resolutions. Figures 5-9 address this issue (by plotting the relative error in dissipation at $\mathrm{t}=.2 \mathrm{sec}$, $.3 \mathrm{sec} ., .4 \mathrm{sec}$., and $.5 \mathrm{sec}$.), and it is encouraging (and expected) that for a fixed resolution, the relative error decreases in the central region of the calculation (i.e., away from the edge of the diffusion front). Also, the region over which reasonable agreement is obtained increases in time for a given resolution. The reason for larger discrepancies at the edges is due to the sharp cutoff in the analytic solution vs. the tail in the numerical solution. If the numerical solution at the edge for the dissipation is $1.0 \mathrm{e}-8$, then this will produce a large relative error, since the analytic solution is 0 (or we could use the floor value of 1.0e-16). Figure 9 shows the effect of increasing the mesh resolution for $t=.4 \mathrm{sec}$. From the figure, it appears as though we are achieving convergence, as the results for 100 and 200 zones seem to collapse nicely in the central region. To make the rate of convergence more quantitative, we can compute error norms from the absolute error at each grid point. For example the Lp-norm is defined as:

$L_{p}(\alpha)=\left[\sum_{i=1}^{N_{\alpha}}\left|\operatorname{error}\left(x_{i}, t\right)\right|^{p} \Delta x_{\alpha}\right]^{1 / p}$,

where $\alpha=1,2,3,4$ corresponds to the four different meshes of 25, 50, 100, and 200 zones. The slope of a log-log plot of $L_{p}(\alpha)$ vs. $\Delta x_{\alpha}$ gives $\mathrm{p}$, the so called order of convergence. Figure 10 shows this information for the dissipation at $\mathrm{t}=.4 \mathrm{sec}$ with the slopes also shown in the legend of the figure. For example, the L2-norm is 1.92, which suggests we are achieving approximately $2^{\text {nd }}$ order convergence. The norms were computed by only considering $\mathrm{x}$-values whose absolute value was less than or equal to .1 to avoid the spurious errors at the edge of the diffusion front. As the spatial derivatives of zone centered fields in Kull reduce to central differences on regular meshes, we should see $2^{\text {nd }}$ order convergence.

\section{References:}

[1] Barenblatt, G. I., Self Similar Turbulence Propagation from an Instantaneous Plane Source, pp. 48-60. In Nonlinear Dynamics and Turbulence, Interaction between Mathematics and Mechanics Series, Pitman.

[2] Memo from Don Eliason to George Zimmerman, May 4, 2004.

[3] Notes from Oleg Schilling, The Barenblatt Model of Diffusion (Turbulent Bursts). 
Dissipation Profiles for Barenblatt Burst (25 Zones)

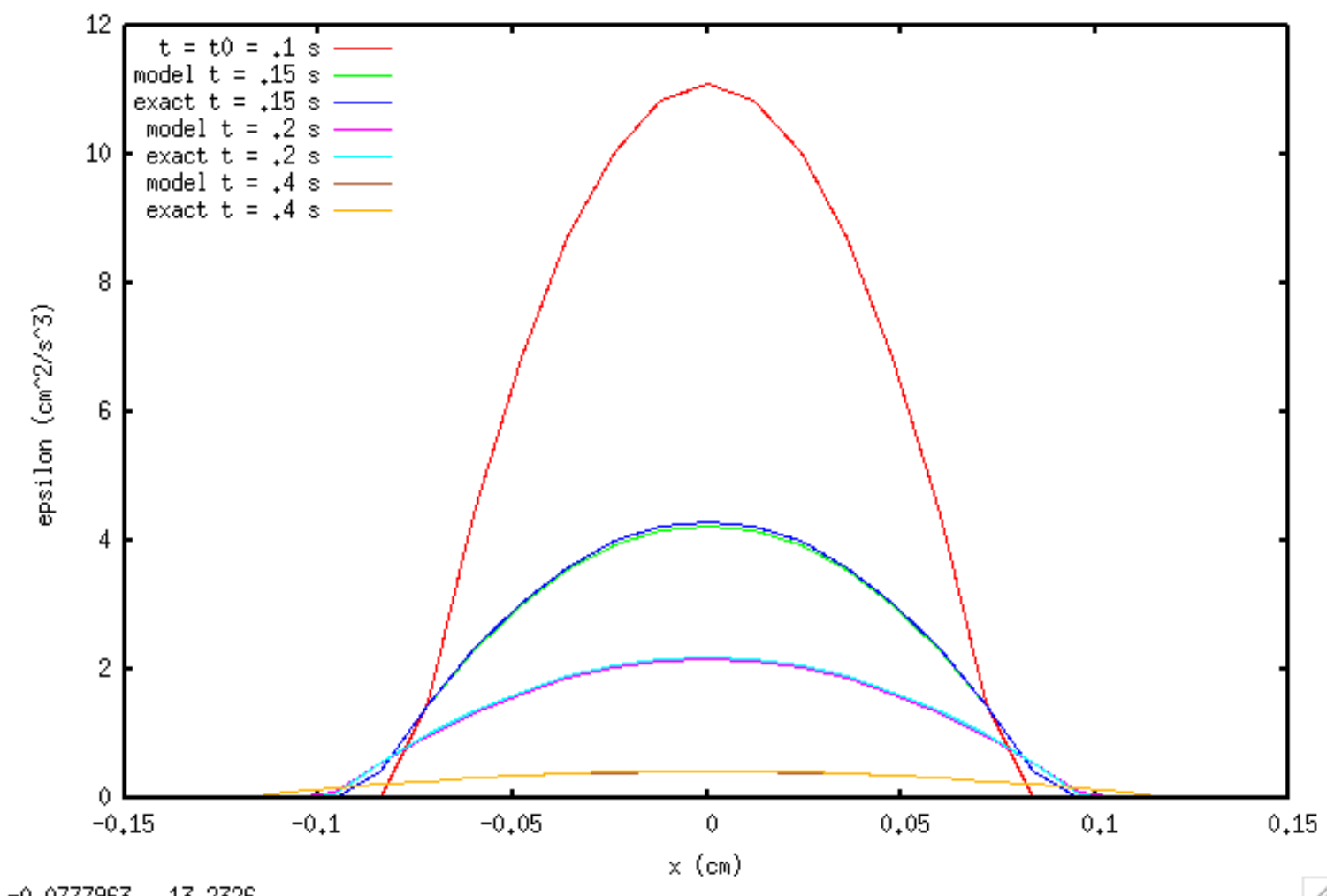

Figure 1 


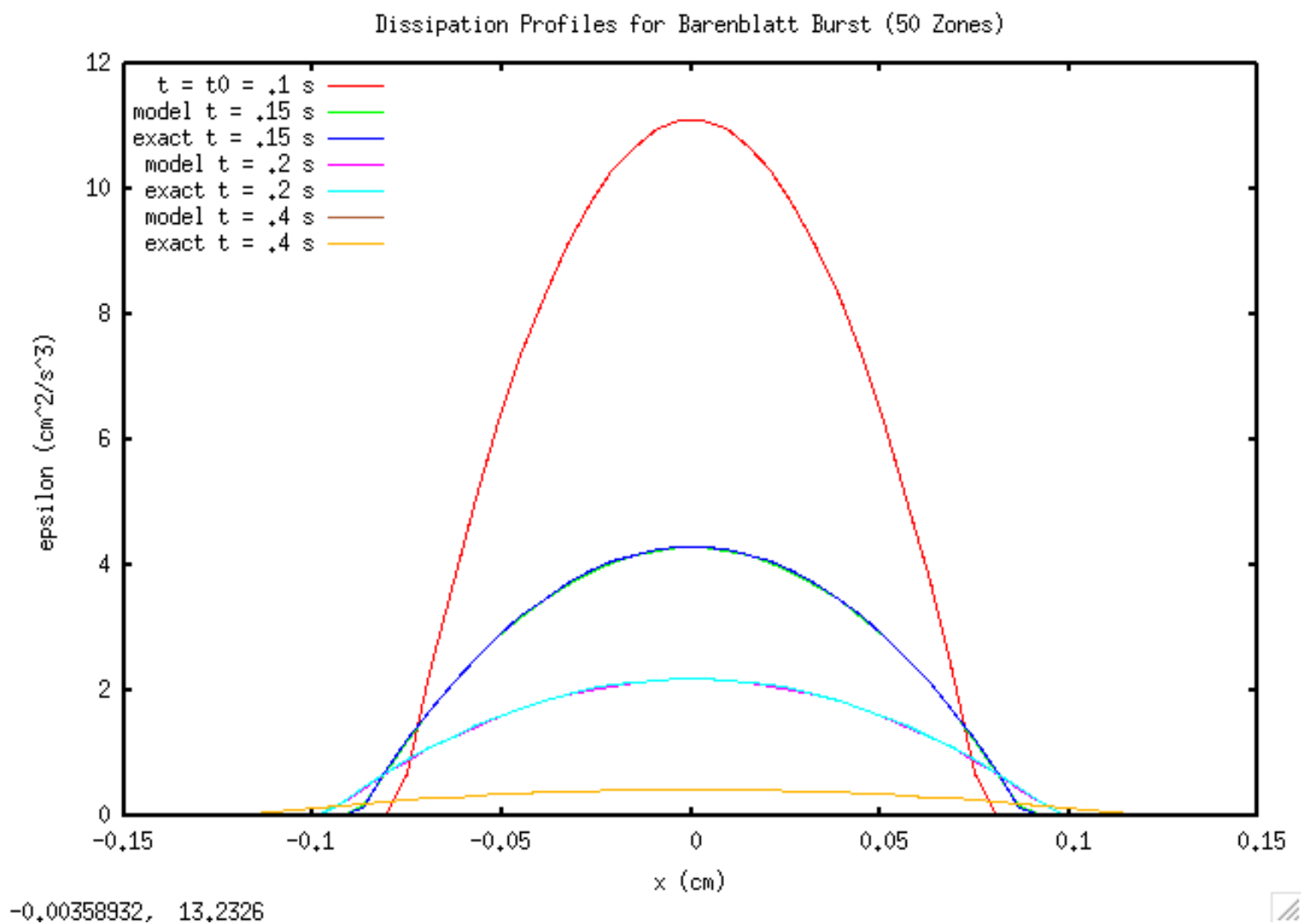

Figure 2 


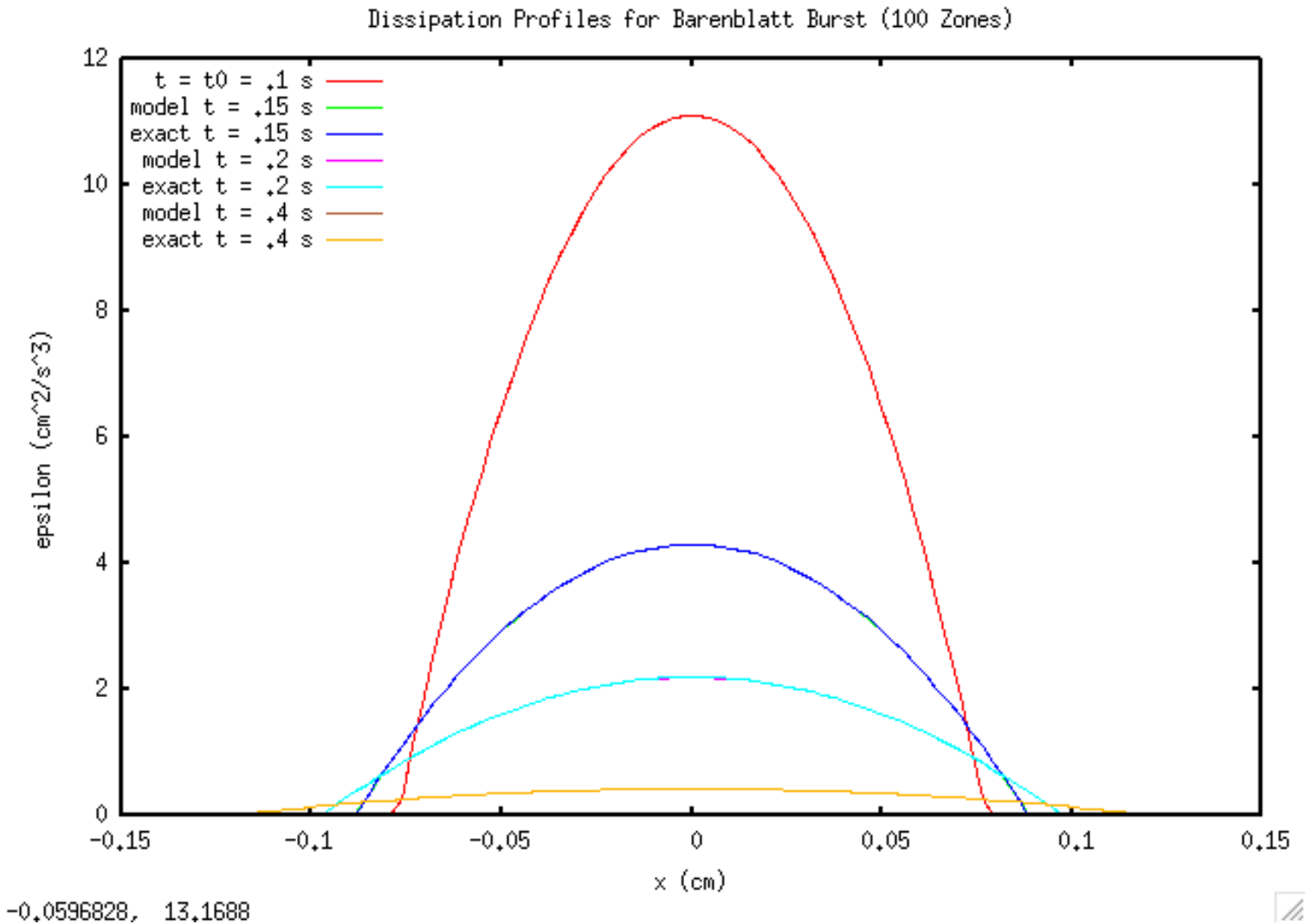

Figure 3 


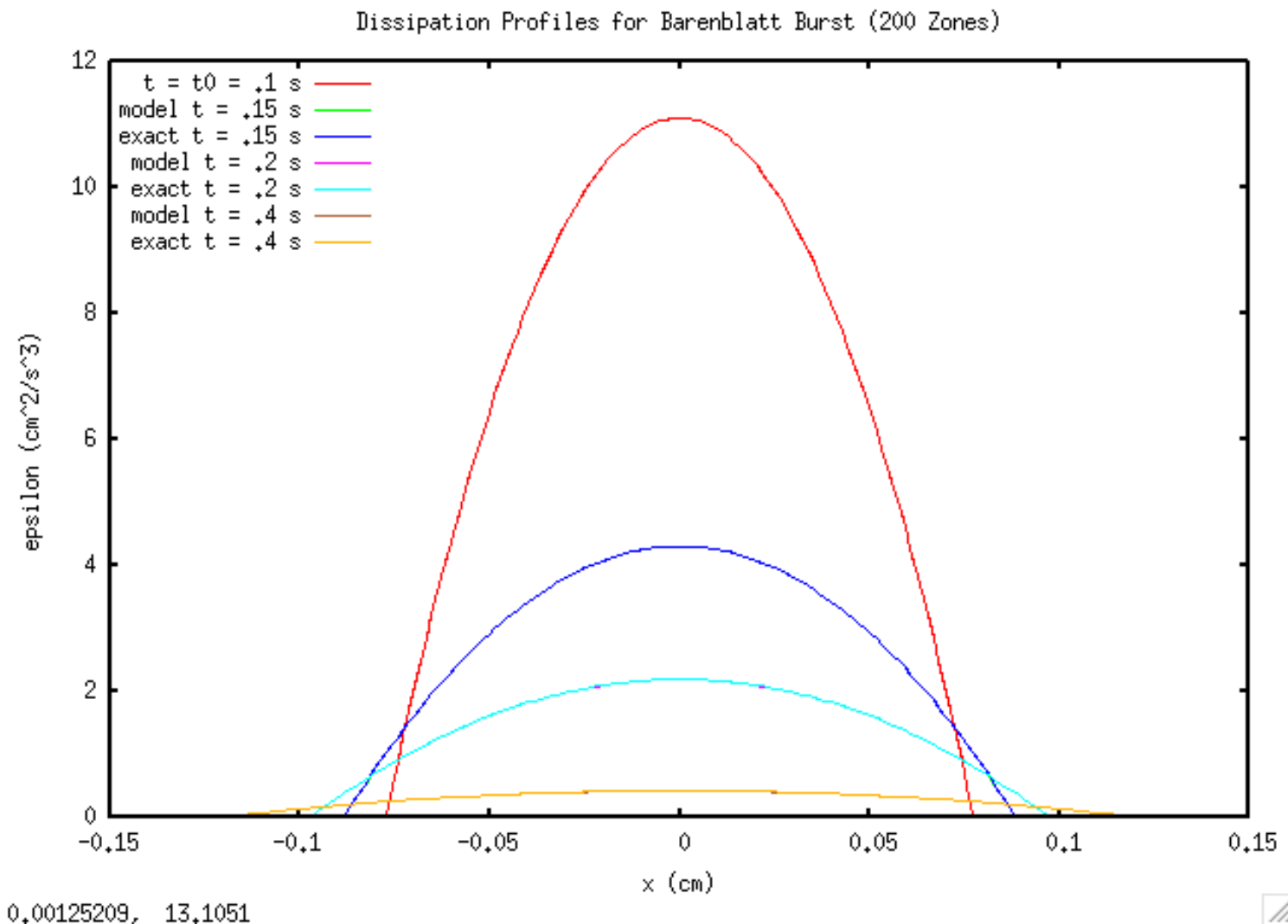

Figure 4 


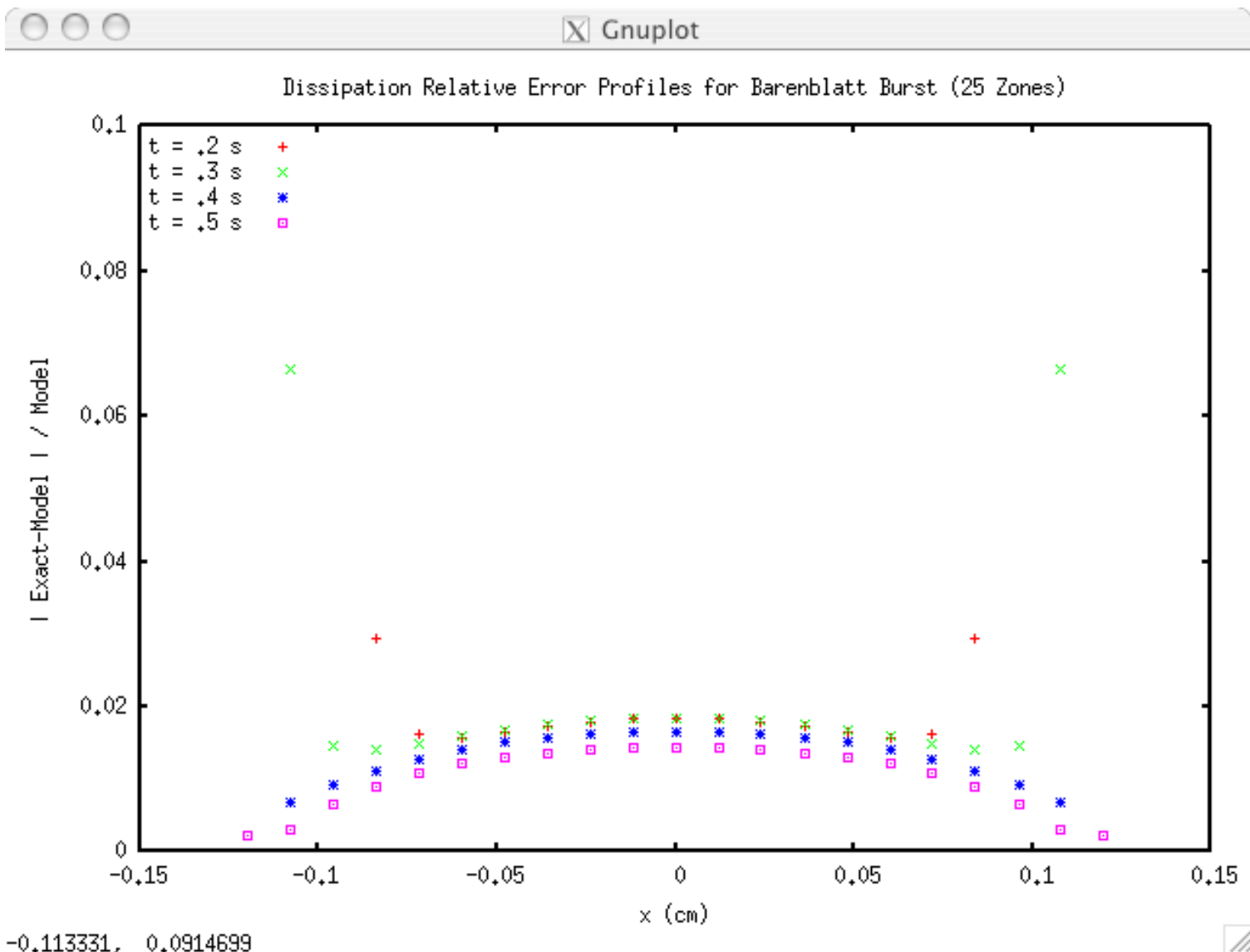

Figure 5 


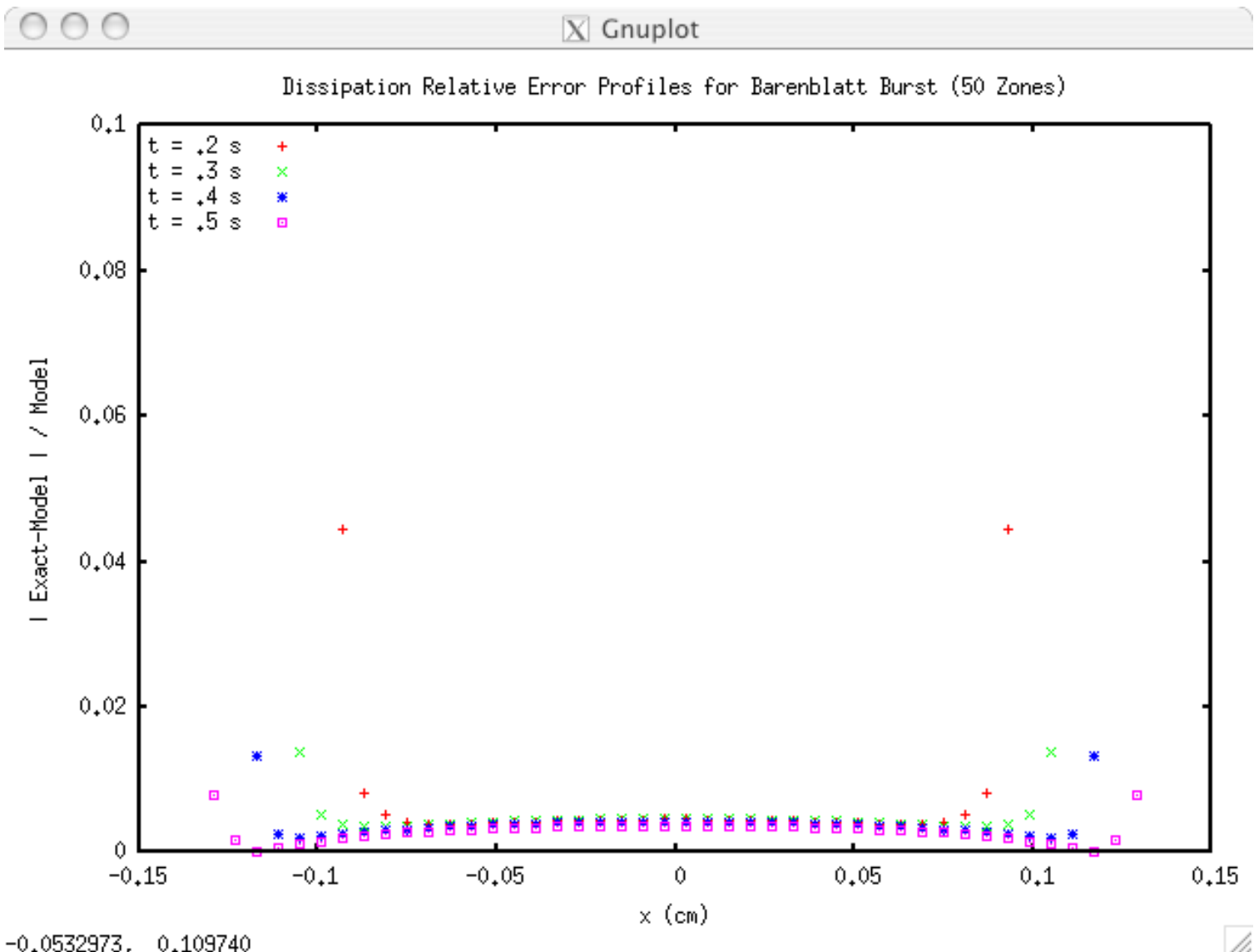

Figure 6 


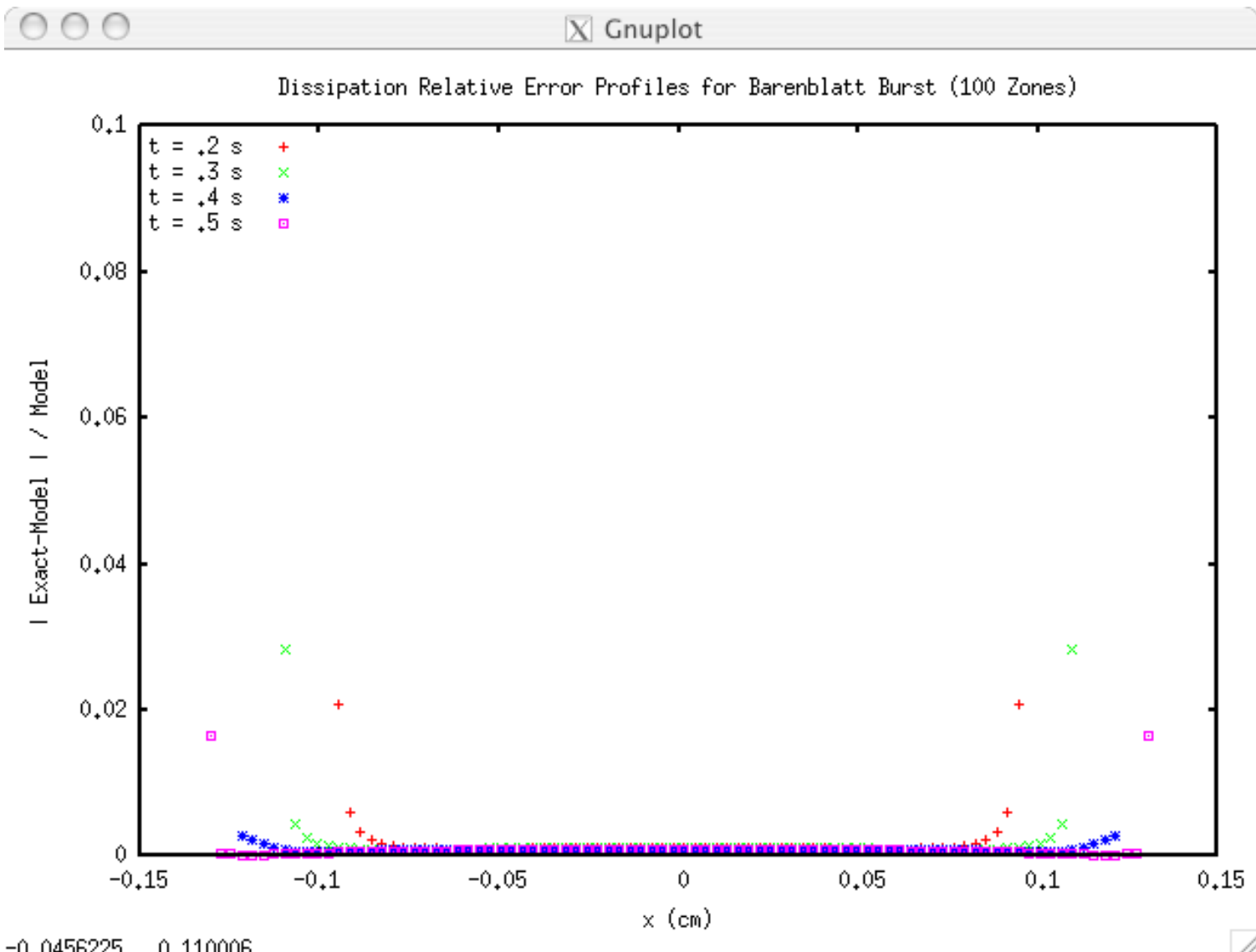

Figure 7 


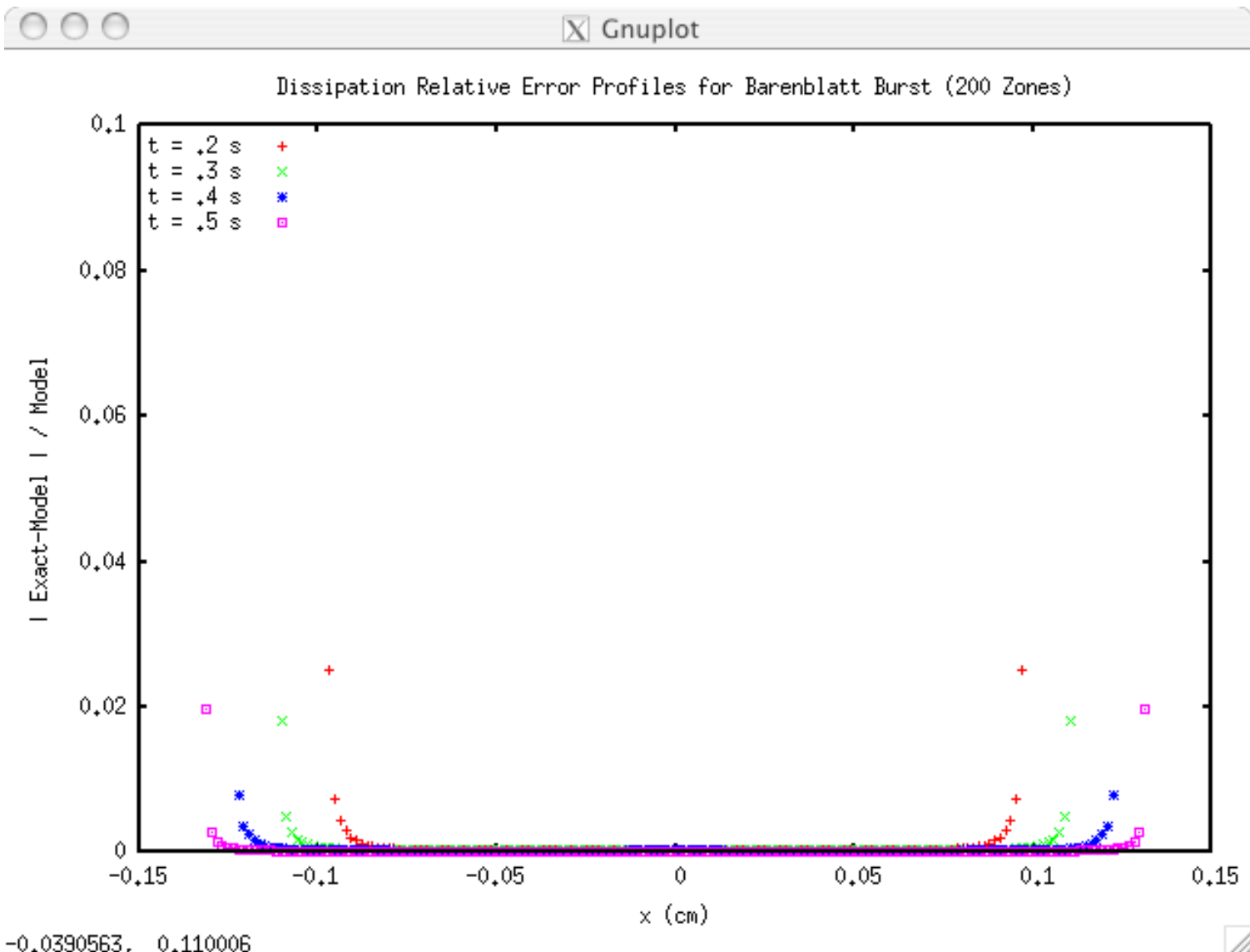

Figure 8 
Dissipation Relative Error Profiles for Barenblatt Burst at $t=.4 \mathrm{~s}$

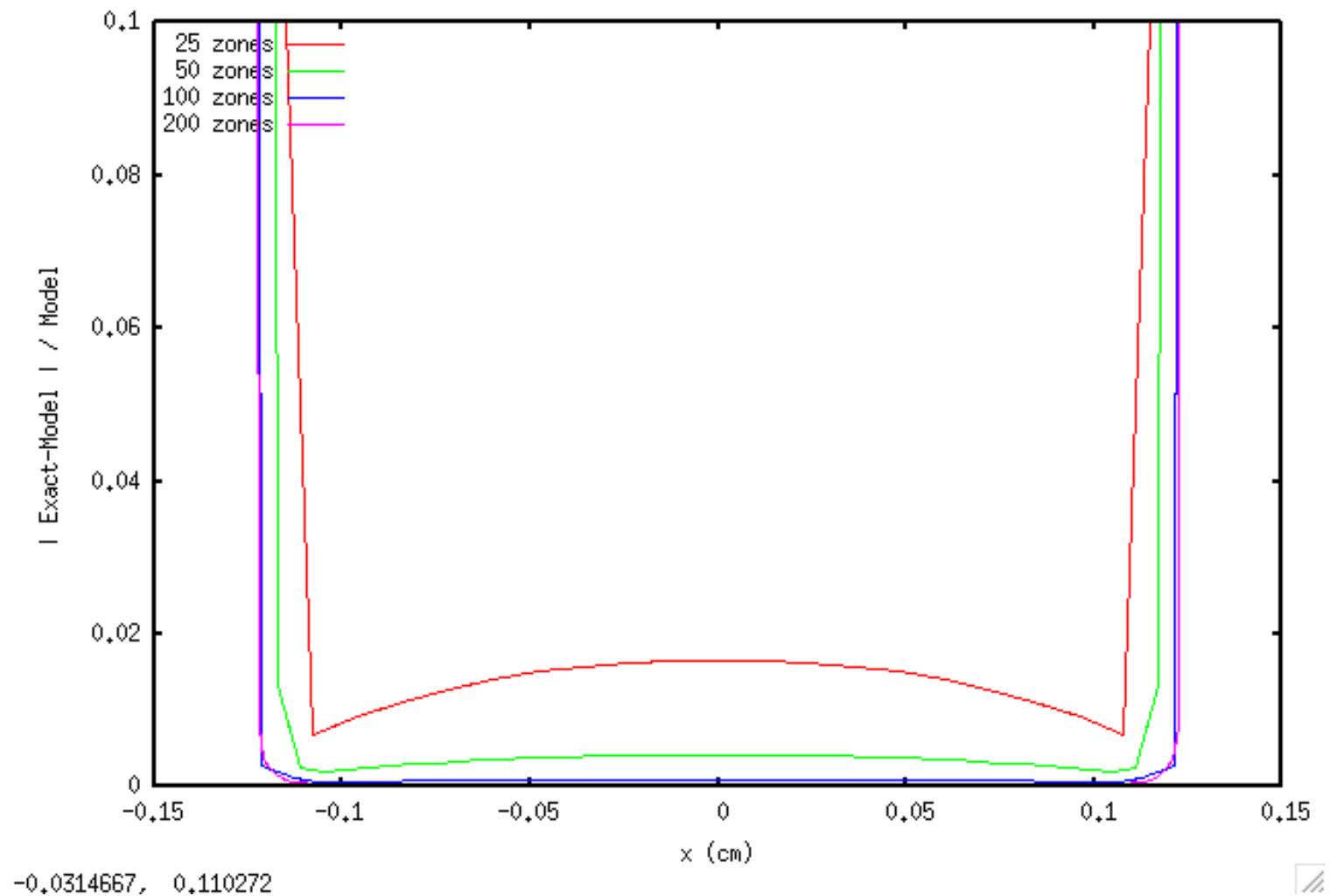

Figure 9 


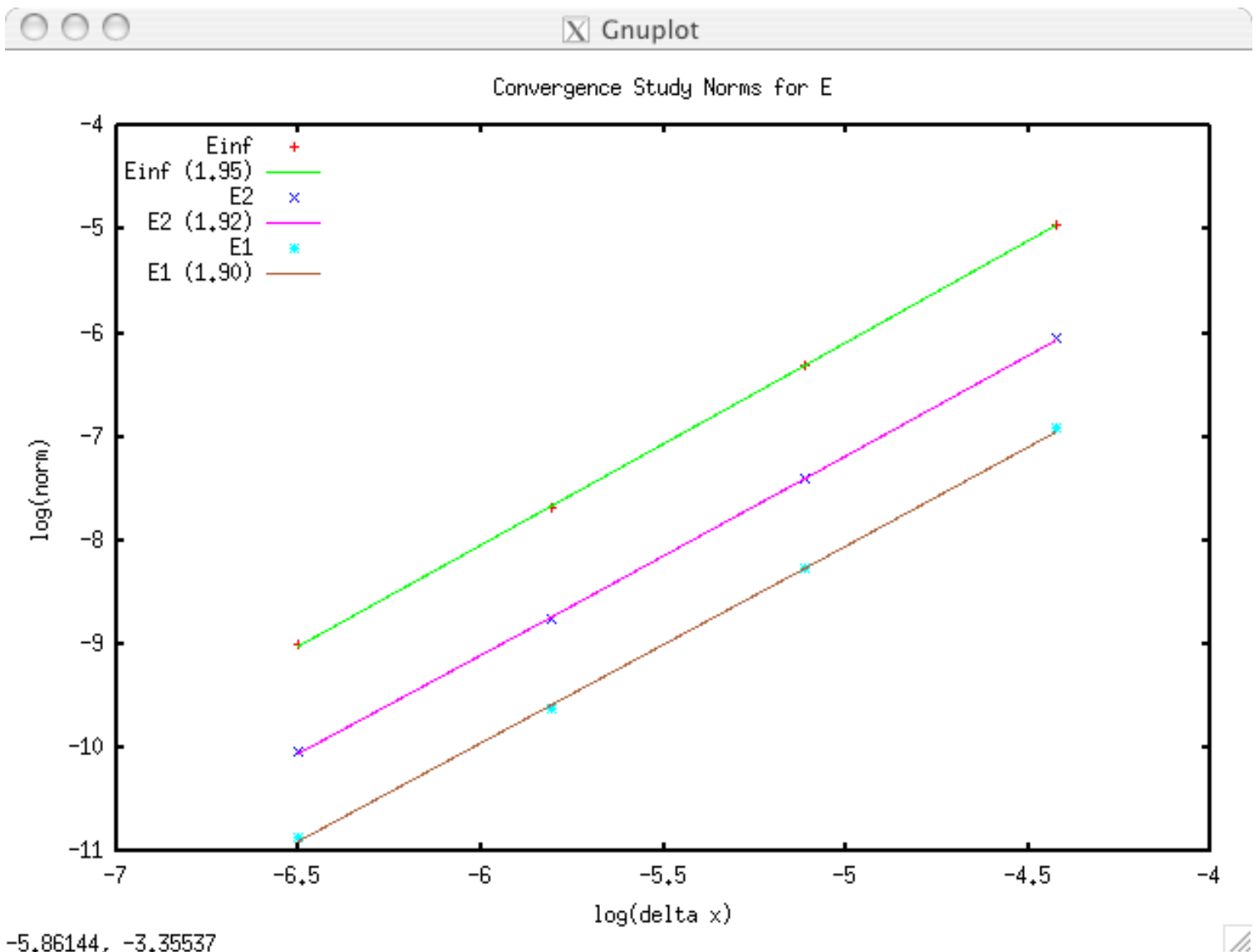

Figure 10 